\title{
Circularly polarised broadband planar lightweight reflectarray with eligible pattern for satellite communications in Ku-band
}

\author{
Pablo Padilla , José Manuel Fernández , José-Luis Padilla , Juan F. Valenzuela-Valdés , Manuel \\ Sierra-Castañer
}

\begin{abstract}
This study presents a lightweight planar patch-array reflectarray at Ku-band for satellite communications. The reflectarray is composed of two separate planar structures: the radiating interface formed by a planar multi-layered broadband patch array, and a phase shifting device formed by $3 \mathrm{~dB} / 90^{\circ}$ couplers. The radiating element is a multi-layered patch structure, designed to provide circular polarisation (CP). Each array cell phase is controlled by open-ended shifting lines of variable length connected to the $3 \mathrm{~dB} / 90^{\circ}$ couplers. The use of this kind of couplers maintains the same CP received/transmitted: the design imposes that if a right-hand CP (RHCP) [or left-hand CP (LHCP)] is received coming from the feeder, the same RHCP (or LHCP) configuration is re-radiated by the reflectarray. Additionally, the feeding horn can be moved in the $x$ or $y$ axes, so that the feeder angular position let re-define the radiation pattern and its pointing direction. Finally, measurements of a lightweight portable planar reflectarray prototype are provided and very good agreement is observed when compared with theoretical results.
\end{abstract}

\section{Introduction}

The operation requirements of satellite communication systems are more and more demanding. The emergence of new commercial applications for satellite communications has forced the development of diverse satellite antenna terminals. Small size antennas $[1,2]$ are expected to afford these challenging requirements, as a result of the development of more powerful satellite transponders. The hardest design constraints are linked to low profile, weight and cost requirements, thus leading to planar printed antenna technology rather than other classic approaches such as reflectors [3, 4]. This way, array antennas such as reflectarrays [5] or artificial lenses (transmitarrays) [6] are employed to provide a similar radiating behaviour to one of the conventional reflector designs, while preserving the strict requirements in terms of size, low profile and weight [7]. In the particular case of a reflectarray structure, the re-definition of the reflected standing wave phase configuration enables the transformation of the original received pattern into new different patterns. This modified radiation pattern depends on the transmission phase and amplitude of the radiated signal of each array radiating element, maintaining the bandwidth requirements $[8,9]$.

Regarding technology, planar antennas are frequently considered in the design of a vast variety of receiving/transmitting terminals due to their attractive characteristics in terms of low profile and weight, low cost, manufacturing accuracy and system integration [10]. In most of the cases, these array-based radiating structures such as reflectarrays mainly include either slot antennas or patch ones for their unitary cell radiating elements [11]. In the case of broadband necessity, the most usual radiating element is the multi-layered patch structure, at the expense of having a thicker structure [12]. Furthermore, there is also the possibility of adding some kind of active behaviour and electronic tuning at each radiating cell, so that the complete array device provides additional reconfigurable functionality [6].

Owing to its robustness against atmospheric effects, circular polarisation (CP) is frequently chosen. This is also the case of the reflectarrays [13-15]. In such structures, the CP can be introduced either by the feeding device (CP horn or linear horn with a polariser) or by the array structure. In the first case, the array structure reflects the incident circularly polarised wave preserving the polarisation; meanwhile, in the second one the linear polarisation coming from the feeder is divided into two orthogonal components at each array cell and a $90^{\circ}$ phase shift is introduced in one of them. This design develops the first option: both the feeder and the array structure have CPs.

In this paper, a circularly polarised broadband high-frequency reflectarray is presented. This device is designed for application in satellite communication systems. This paper is organised as follows: Section 2 presents the main characteristics of antenna structure and the design process. Section 3 is devoted to prototypes and their measured results. Finally, conclusions are drawn in Section 4.

\section{Reflectarray design}

In this section, the planar antenna structure and the design of the different antenna layers are provided. The system requirements are presented in Table 1 .

The reflectarray consists of a feeding horn that illuminates a $240 \times 240 \mathrm{~mm}^{2}$ planar structure. The planar structure is made of three different layers. Two layers are devoted to the radiating elements (two-layered stacked patches) and an additional layer is devoted to locate the shifting circuits. The radiating layers and the shifting one are separated by a ground plane. The complete design configuration is provided in Fig. 1, and the different layers are shown in Fig. 2, and described in what follows:

- Shifting layer: It is formed by a two-dimensional (2D) array of $3 \mathrm{~dB} / 90^{\circ}$ couplers, printed over the upper side of a Neltec substrate (NY9217, $\left.\varepsilon_{\mathrm{r}}=2.17, h=0.508 \mathrm{~mm}, \tan \delta=0.0002\right)$. The other substrate side contains a copper ground plane with circular holes without copper (radius $r_{\text {hole }}=1.5 \mathrm{~mm}$ ) around the 
Table 1 Reflectarray antenna specifications

\begin{tabular}{lcc}
\hline Parameter & Value & Units \\
\hline frequency bands & $11.5-12.5$ & $\mathrm{GHz}$ \\
polarisation & LHCP/RHCP & - \\
AR & $<2.5$ & $\mathrm{~dB}$ \\
3 dB beamwidth & 7 & $\circ$ \\
sidelobe level & $<-16$ & $\mathrm{~dB}$ \\
cross-polarisation & $<-25$ & $\mathrm{~dB}$ \\
gain & $>25.5$ & $\mathrm{dBi}$ \\
directivity & $\sim 27$ & $\mathrm{dBi}$ \\
radiation efficiency & $>65$ & $\%$ \\
VSWR & $1.4: 1(-15.6 \mathrm{~dB})$ & - \\
size (planar structure) & $240 \times 240 \times 3.524$ & $\mathrm{~mm}$ \\
array separation & $0.55 \lambda$ & $\mathrm{mm}$ \\
focal distance & 210 & $\mathrm{~mm}$ \\
weight & $<825$ & $\mathrm{~g}$ \\
\hline
\end{tabular}

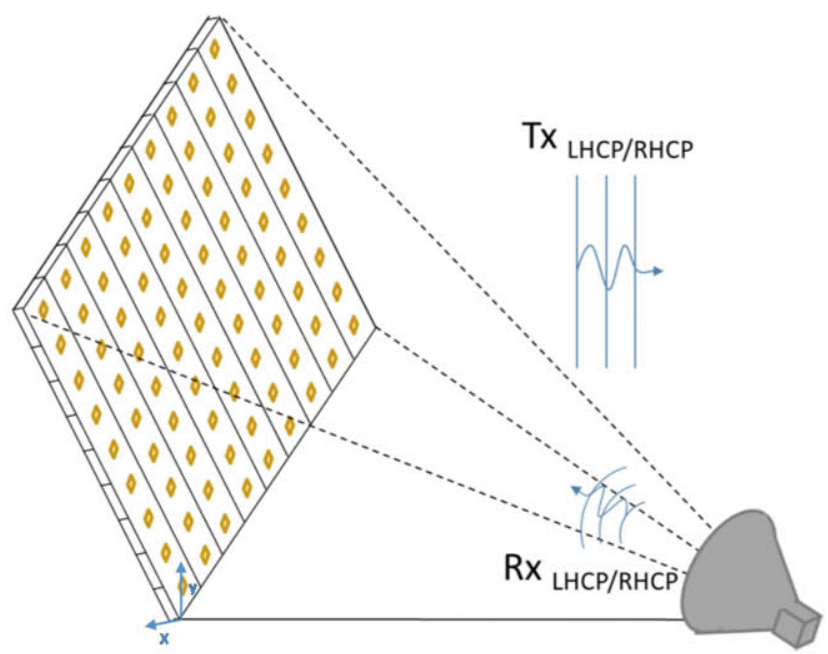

Fig. 1 Complete reflectarray antenna (planar structure plus feeding horn)

location of the vias (radius $r_{\mathrm{via}}=0.6 \mathrm{~mm}$ ) that connect this layer with the next one.

- Lower patch layer: It consists of a 2D array of square patches over a Neltec substrate with the same properties and dimensions than the shifting layer. The other side remains without any copper and will be in contact with the copper ground plane of the shifting layer. The patches are connected with the $3 \mathrm{~dB} / 90^{\circ}$ couplers of the shifting layer by means of the metallic vias.

- Upper patch layer: It consists of an additional 2D array of square patches over another Neltec substrate with the same properties and dimensions than the previous ones. The other side has no copper. The interaction between the lower-layer patch array and the upper one is obtained by inductive coupling. These two patch layers are additionally separated by a foam layer of height $h_{\text {Foam }}=2 \mathrm{~mm}$.
Thus, the profile dimensions are $d=3 h+h_{\text {Foam }}=3.524 \mathrm{~mm}$. Regarding the weight of the reflectarray radiating multi-layered structure, its weight is $<218 \mathrm{~g}$ (NY9217 substrate $2.23 \mathrm{~g} / \mathrm{cm}^{3}$ and foam $0.2 \mathrm{~g} / \mathrm{cm}^{3}$ ). The lightweight of this antenna is a general property of planar printed circuit architecture.

Although there are some single-layer radiating elements such as split ring, double split ring, $C$ ring etc. that are suitable for $\mathrm{CP}$ arrays providing a broadband functioning, they are quite dependent on the particular configuration they are designed for. For example, an element designed to have a good $\mathrm{CP}$ behaviour maybe not suitable for linear polarisation. Our design is much more flexible because the reflectarray functioning in terms of polarisation depends only on the feeder and its polarisation. In fact, our design is valid for left-hand CP (LHCP), right-hand CP (RHCP) or a linearly polarised feeder.

The feeding horn is a $12 \mathrm{GHz}$ circularly polarised circular horn, located at the centre of the horizontal axis (x-axis) and with an offset in the vertical axis ( $y$-axis), the same configuration than the one depicted in Fig. 1 (the offset angle of the feeder is $28^{\circ}$ ). The complete planar structure is, thus, organised as an array of unitary cells. Each cell contains a stacked-patch radiating element over a ground plane, connected to a $3 \mathrm{~dB} / 90^{\circ}$ shifting circuit by a metallic via. The reason of using a $3 \mathrm{~dB} / 90^{\circ}$ coupler is to be able to deal with $\mathrm{CP}$. The working principle of the unitary cell implies: (i) the reception of the signal in the radiating part, (ii) its reflection in the shifting layer and (iii) its re-radiation in the radiating structure. The phase shifter consists of the $3 \mathrm{~dB} / 90^{\circ}$ coupler with ports 3 and 4 connected to two identical circuit lines of length $l$ ended in an open circuit, so that the signals introduced in ports 1 and 2 are guided toward ports 3 and 4 , reflected at the open circuits and redirected to ports 2 and 1, respectively, with twice the phase shift provided by the circuit lines of length $l$ (see Fig. 3 for port location). In this case, the two orthogonal polarisation components of the $\mathrm{CP}$ are received with the same amplitude and a $90^{\circ}$ phase difference, and are exposed to the same phase shifting at each unitary cell of the array. The use of $3 \mathrm{~dB} / 90^{\circ}$ couplers preserves the same kind $\mathrm{CP}$ received, either LHCP or RHCP. The possibility of keeping the same polarisation adds flexibility to the design, and it depends on the designer criterion, and also the feeder horn features. Note that, depending on the design requirements, it could be as important to keep the polarisation or to maintain the usual polarisation change.

To complete and validate the designs, Computer Simulation Technology (CST) Studio Suite simulation software has been used, for both the unitary array cell and the complete array itself. Fig. 3 depicts the simulation model for the unitary cell. Although the initial reflectarray specifications fixed a bandwidth of at least 1 $\mathrm{GHz}$ over the working frequency of $12 \mathrm{GHz}$, the design provides a $-10 \mathrm{~dB}$ bandwidth of $2.1 \mathrm{GHz}(17.5 \%)$. The dimensions of the radiating elements and the couplers are provided in Table 2 . Both the upper and lower patches are square-shaped with different side lengths. Additionally, the lower patches have two bevelled corners to reinforce the cell matching. More details about cell design and performance, array definition and design concerns can be found at [16].

Once the unitary cell is designed, the complete reflectarray configuration is defined, in order to convert the quasi-spherical pattern of the feeder into a plane wave. Additionally, angular mobility is added to the feeder supporting in the principal axes: $\theta_{x}$

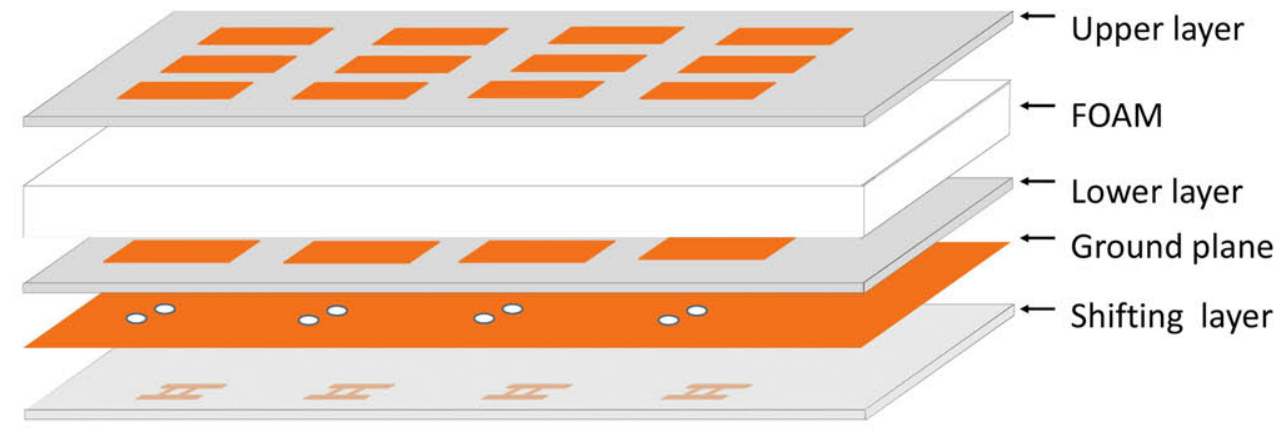

Fig. 2 Details of the planar structure 

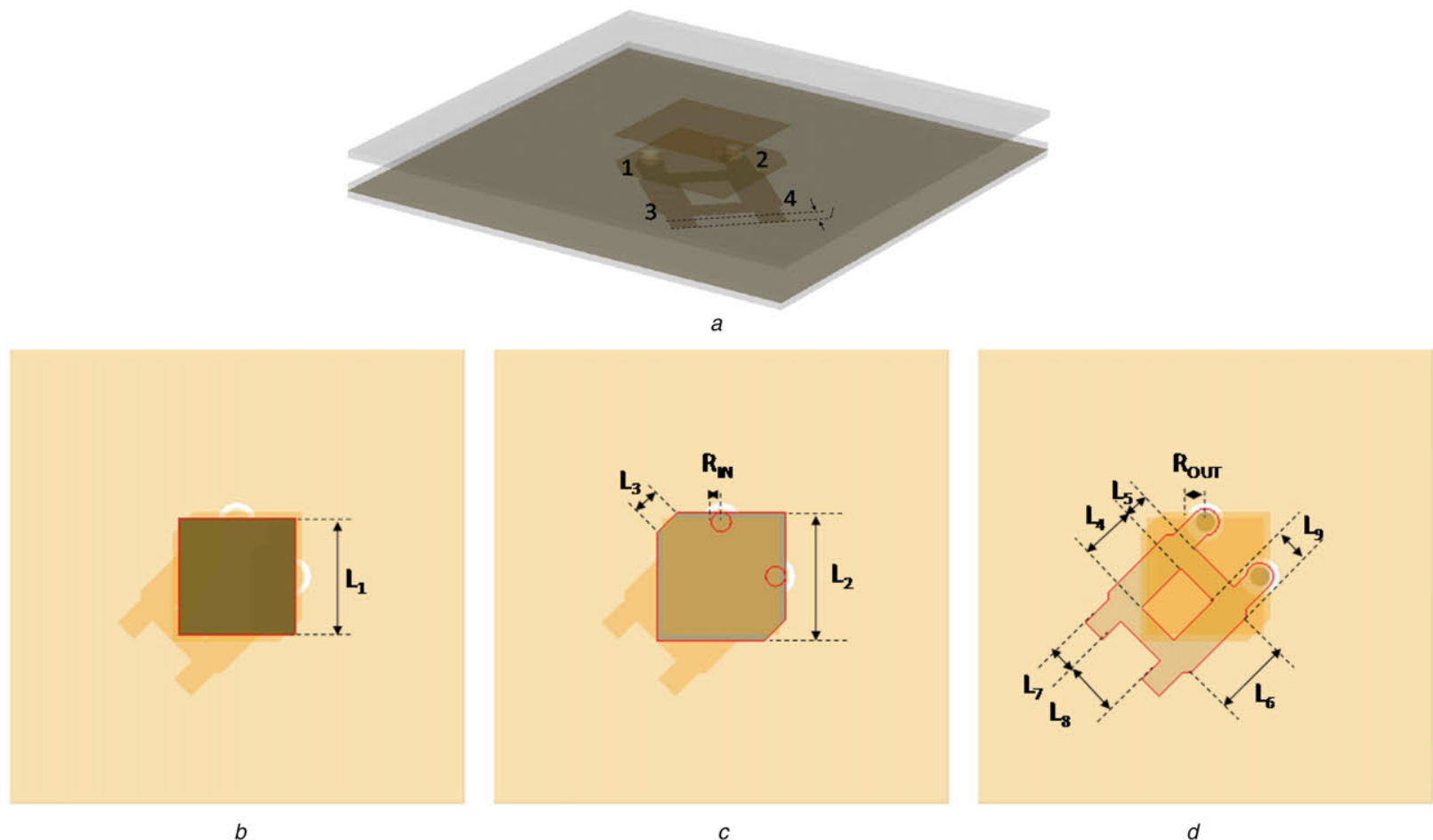

Fig. 3 Complete cell

(a) Array simulation model, (b) Dimension parameters

Table 2 Cell design parameters and dimensions

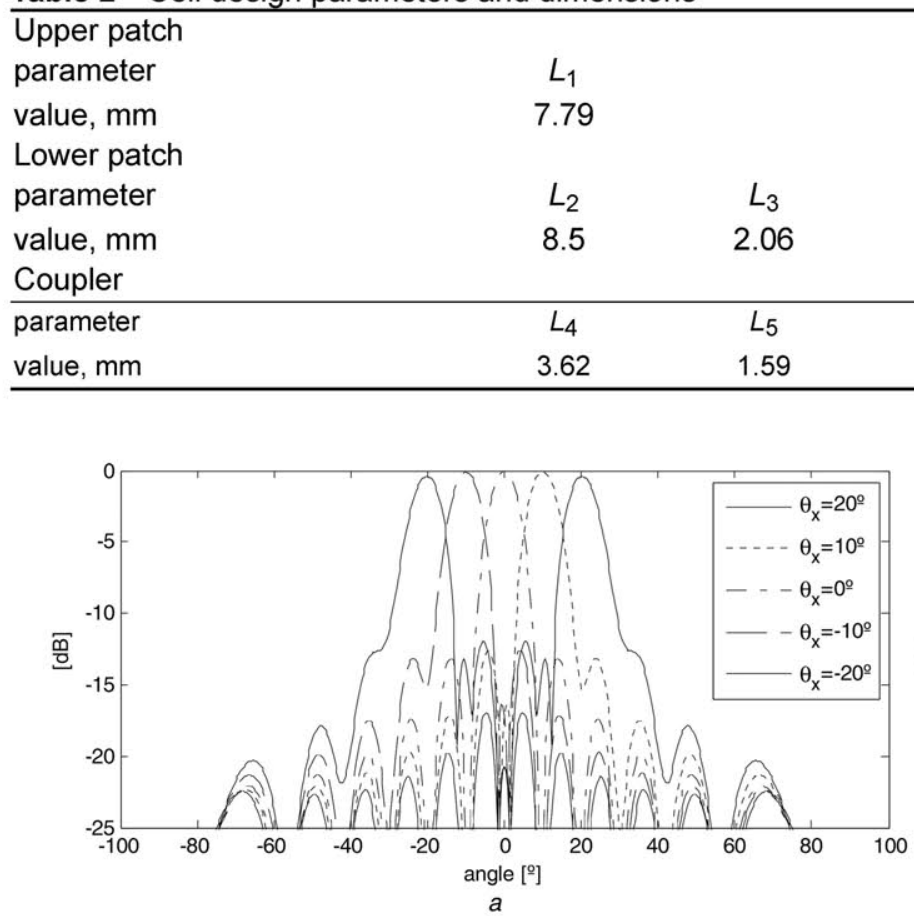

Fig. 4 Pattern configuration according to

(a) $\left[\theta_{x}, 0\right],(b)\left[0, \theta_{y}\right]$

and $\theta_{y}$. Although 3D movement can be added with convenient gyroscopic joints, in this case only one component can be moved at each time, that is, $\left[\theta_{x}, 0\right]$ or $\left[0, \theta_{y}\right]$. The array structure is conceived to permit the feed angular movement along the $x$ and $y$ axes with the intention of preserving the pattern shape but with a variation in the steering direction. Fig. 4 provides some examples of the pattern configuration according to different values of $\theta_{x}$ and $\theta_{y}$. As it can be noted, the angular variation introduces a small degradation in the pattern configuration and, as a consequence, a small reduction in the antenna gain. This gain degradation increases as the angular variation is increased, as it is identified in the example in Fig. 4

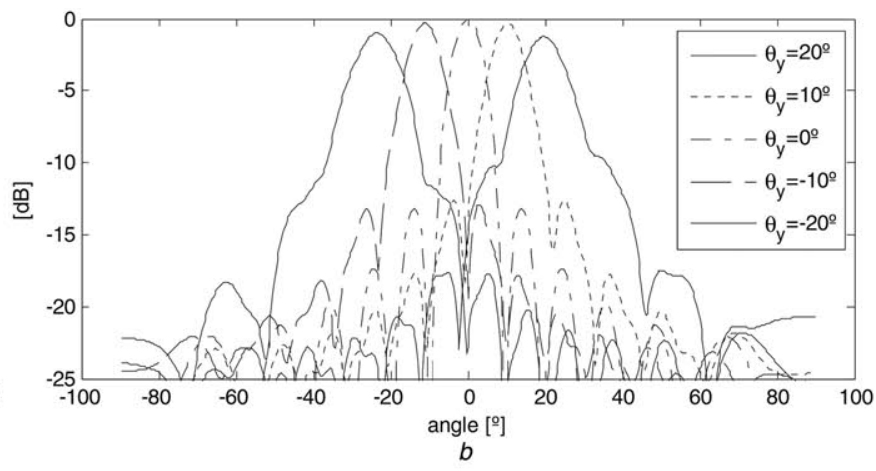

(for example, for the $\theta_{x}$ variation, the gain reduction is of $0.1 \mathrm{~dB}$ in the case of $\pm 10^{\circ}$ variations and $0.4 \mathrm{~dB}$ in the case of $\pm 20^{\circ}$ variation). Regarding the shape degradation due to the feed offset variation, it can be also compensated [17].

\section{Antenna prototype and measurement results}

Once the desired phase distribution has been calculated for the reflectarray feeder position $\left(x_{0}, y_{0}, z_{0}\right)$, the manufacture of the different layers is accomplished. The elements are printed over the different substrates by means of conventional copper photolithography etching process with copper of $35 \mu \mathrm{m}$ thickness. 


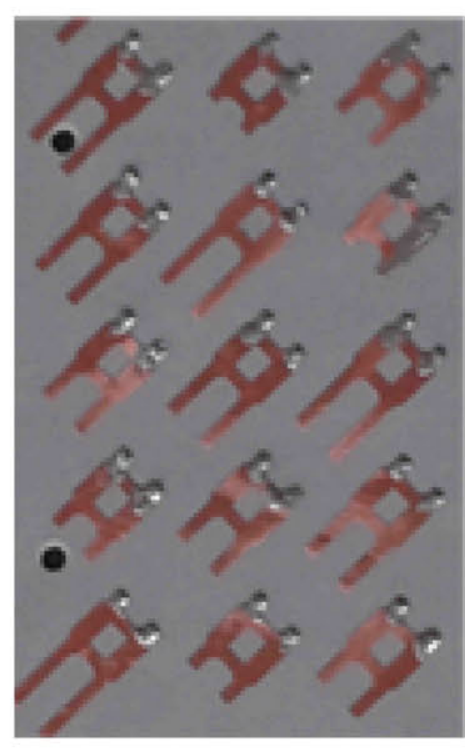

a

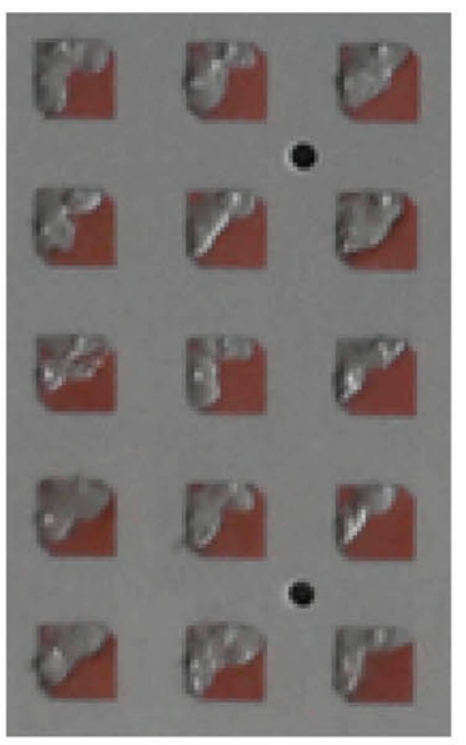

$b$

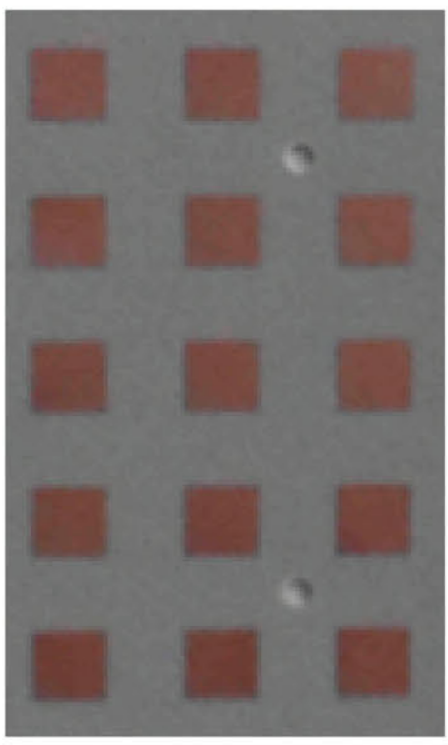

$c$

Fig. 5 Zoom view of the elements in the three different layers of the prototype (a) Shifters, (b) Lower patches, (c) Upper patches

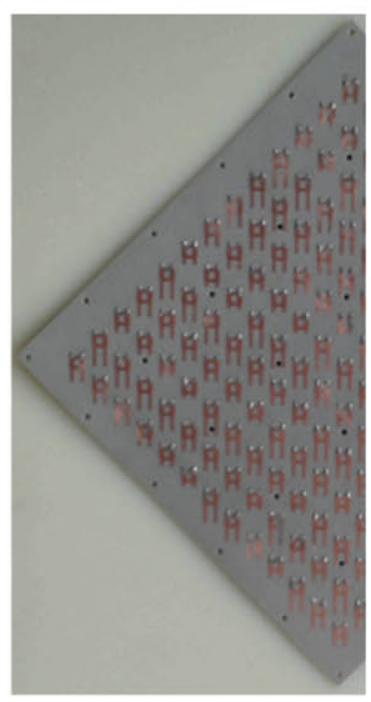

a

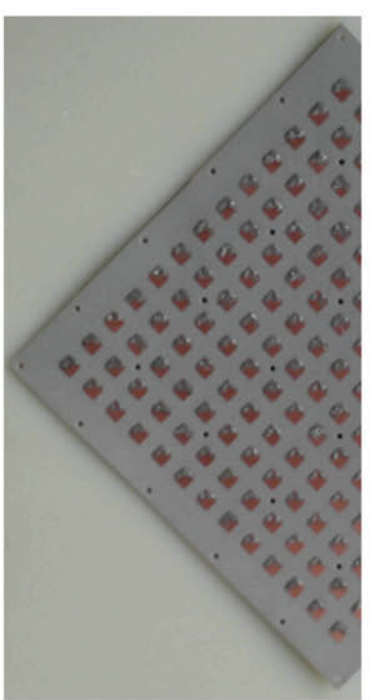

b

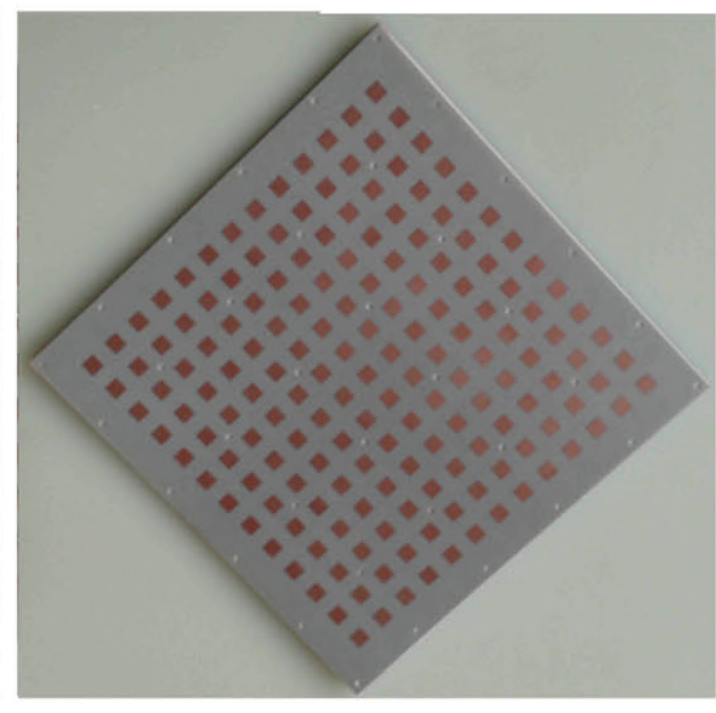

c

Fig. 6 Three different layers of the prototype (a) Shifters, (b) Lower patches, (c) Upper patches

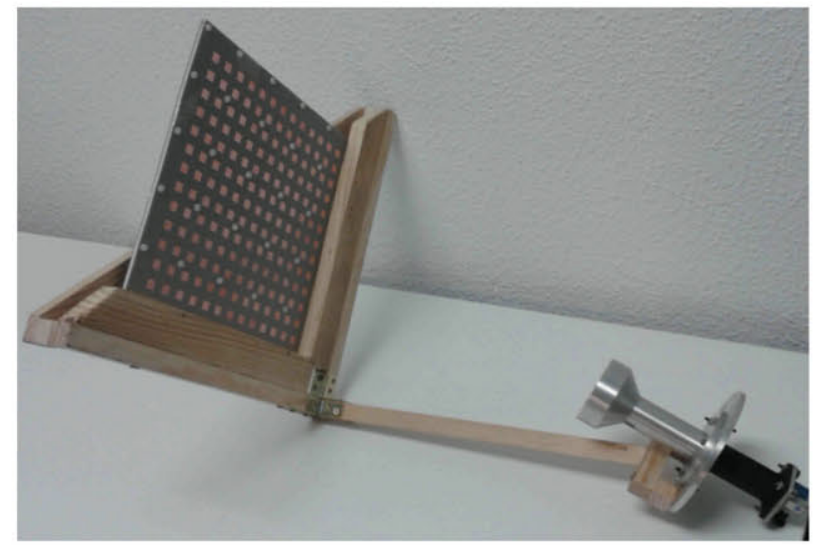

Fig. 7 Complete prototype mounted, with feeding horn

Fig. 5 presents zoom-in views of the different layers: shifters, lower patches and upper patches. The lower patches are connected to their corresponding shifters with welded metallic vias. Fig. 6 provides the three different layers of the planar structure. Fig. 7 provides an image of the complete prototype already mounted and

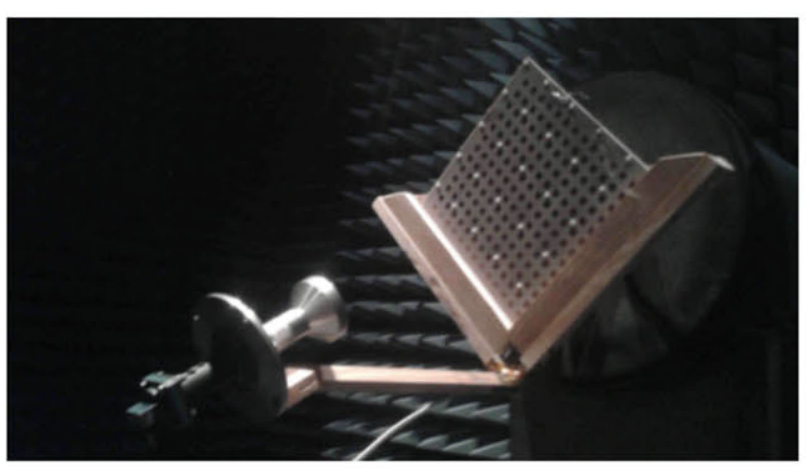

Fig. 8 Measurement setup for the prototype measuring process in the anechoic chamber at the Technical University of Madrid

calibrated, ready for its validation and measuring in anechoic chamber.

The prototype measurement setup in the anechoic chamber is provided in Fig. 8. Measurements regarding antenna radiation patterns and axial ratio (AR) are provided in Figs. 9 and 10. 


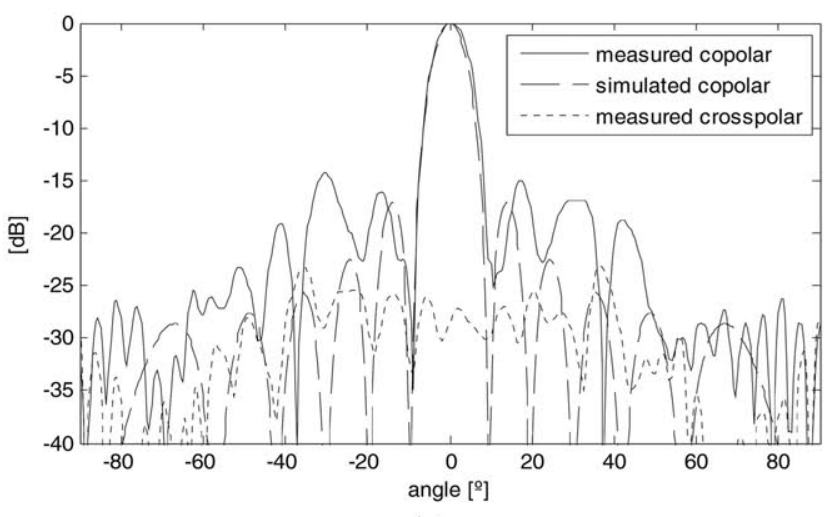

:a

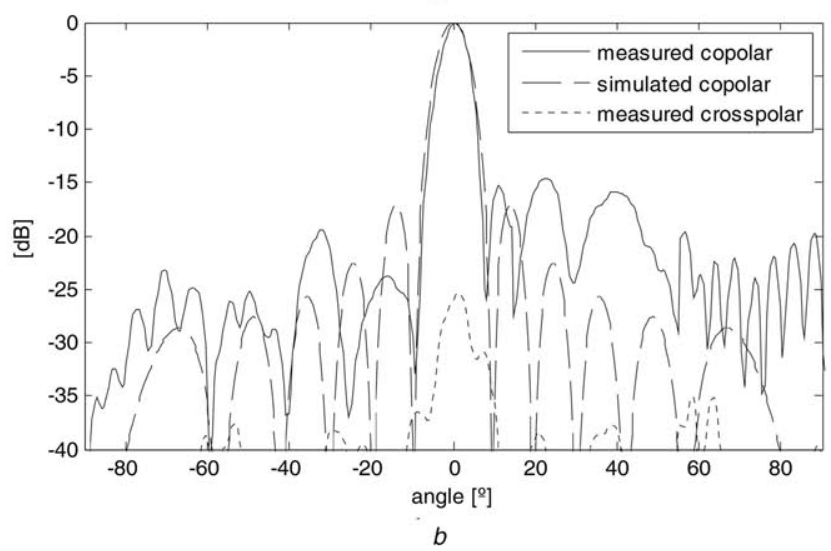

Fig. 9 Radiation pattern of the reflectarray antenna prototype, measurements versus simulations, at $12 \mathrm{GHz}$, for the main axes (a) $\varphi=0^{\circ}$ ( $x$-axis), (b) $\varphi=90^{\circ}$ (y-axis)

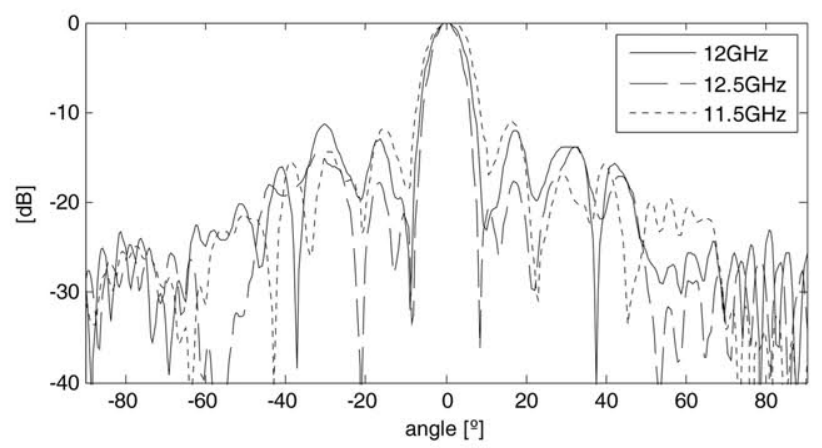

Fig. 10 Radiation pattern of the reflectarray antenna prototype, at 11.5, 12 and $12.5 \mathrm{GHz}$, for the main axis $\varphi=0^{\circ}$ (x-axis)

The reflection coefficients fulfil the initial specifications, $S_{11}<$ $-20 \mathrm{~dB}$ in the whole bandwidth. The radiation efficiency is over $70 \%$. The antenna pattern measurements are carried out in the principal planes $\left(\varphi=0^{\circ}, \varphi=90^{\circ}\right)$ in Cartesian plot as it can be seen in Figs. 9 and 10. The co-polar (CP)-cross-polar ratio is better than $26 \mathrm{~dB}$ in the broadside direction and sidelobe levels are below 16

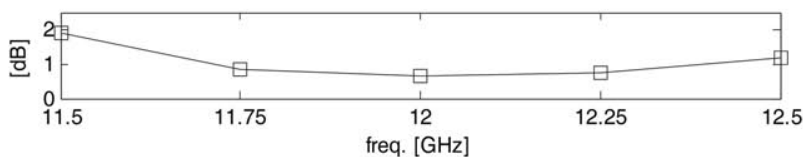

Fig. 12 AR of the antenna prototype

$\mathrm{dB}$. The measured gain value at the central frequency $(12 \mathrm{GHz})$ is $26.5 \mathrm{dBi}$ and the values at the edge frequencies, 11.5 and 12.5 $\mathrm{GHz}$, are 25.7 and $25.6 \mathrm{dBi}$, respectively, which fulfils the requirement in terms of gain bandwidth in the entire working band $(>1 \mathrm{GHz}$ of $1 \mathrm{~dB}$ gain bandwidth). Fig. 11 provides the gain, radiation efficiency and aperture efficiency values of the antenna measurements in the frequency range [11.5 GHz, $12.5 \mathrm{GHz}]$, for five frequency values. Fig. 12 provides the AR: an AR lower than $1.95 \mathrm{~dB}$ is measured for the reflectarray antenna.

The discrepancies between measured co-polar and simulated one are due to manufacturing tolerances that yield small phase errors at each array cell. The manufacturing laboratory tolerances guaranteed a tolerance lower than $\pm 125 \mu \mathrm{m}$ in the circuit etching. It has been computed that a uniformly distributed random $\pm 5^{\circ}$ error ( $125 \mu \mathrm{m}$ in delay line lengths) in simulation provides a sidelobe level quite similar to the one measured.

As it can be noted, there are some issues that become significant contributions in this paper. First of all, the design presents very good performance results with a very simple and affordable design and manufacturing process, with a quite good agreement between measurements and expected outcomes (radiation pattern, sidelobe levels, beamwidth etc.). Second, the structure let vary the reflectarray steering direction by means of a change in the feeding horn direction of incidence: although in this particular prototype only one component can be moved at each time, a 3D movement could be added with a convenient gyroscopic joint. Third, though each single element in the unitary reflectarray cell maybe considered as an already known element, the particular way in which they are combined is also an interesting contribution in the document: as examples the fact of transforming a $3 \mathrm{~dB} / 90^{\circ}$ coupler into a dual CP phase shifter, and the preservation of the same kind of CP received, either LHCP or RHCP, among others.

\section{Conclusion}

This paper presents a circularly polarised broadband planar lightweight reflectarray with eligible radiating pattern for satellite communication systems in Ku-band. The reflectarray is based on a lightweight three-layer structure composed by multi-layered patches in the radiating interface and open-ended $3 \mathrm{~dB} / 90^{\circ}$ couplers acting such as phase shifters in the phase control interface. The phase configuration in the shifting interface is arranged, so that the quasi-spherical pattern of the feeding horn is translated into a plane wave. Angular mobility $\left(\theta_{x}, \theta_{y}\right)$ in the feeding horn position is introduced, so that it is possible to re-define the $3 \mathrm{D}$ radiating pattern pointing direction mechanically. Additionally, the use of open-ended $3 \mathrm{~dB} / 90^{\circ}$ couplers let preserve the original polarisation of the feeder. The design is validated by manufacturing a reflectarray prototype according to the design parameters and values. The reflectarray radiating features are analysed and a proper performance is pointed out. The experimental results also

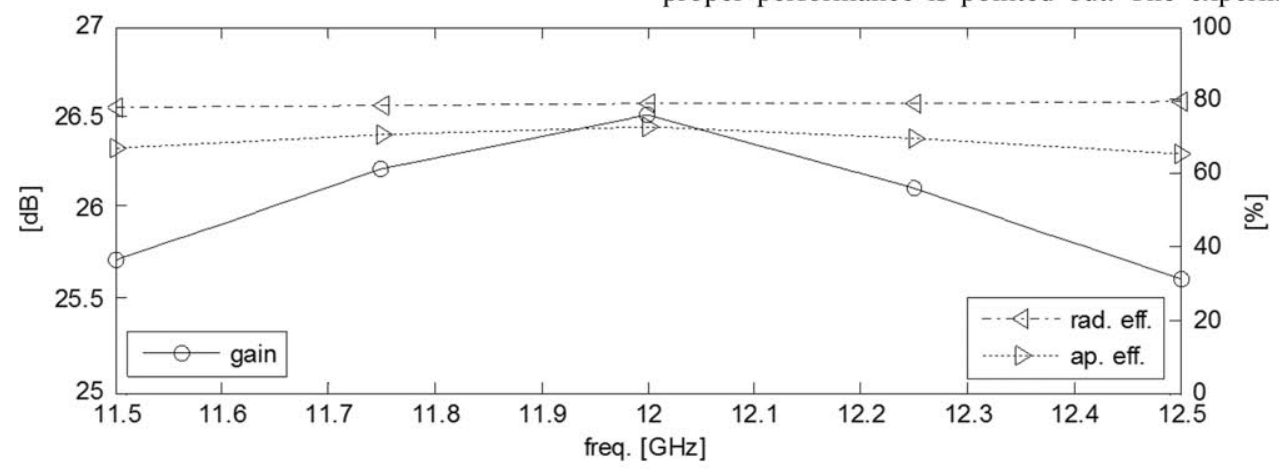

Fig. 11 Gain, aperture efficiency and radiation efficiency of the antenna in the frequency range 
demonstrate high radiation and aperture efficiencies. The agreement of the experimental characterisation with the simulations validates the analysis, design and fabrication process of this reflectarray antenna.

\section{Acknowledgments}

This work has been supported by the TEC2014-55735-C3-1-R, UNGR15-CE-3311 and TIN2016-75097-P projects of the Spanish National Program of Research, Development and Innovation. The simulations in this work have been carried out using CST Studio Suite under a cooperation agreement between the Computer Simulation Technology (CST) and the Technical University of Madrid. Substrates used in the prototypes were kindly provided by NELTEC.

\section{References}

[1] Evans, J.V.: 'Satellite systems for personal communications', Proc. IEEE, 1998, 86, (7), pp. 1325-134

[2] Salas, M.A., Garcia, A., Mora, I., et al.: 'Satellite communications, chap. 7 'New antenna arroy architectures for satellite communication', (InTech Open Access Publisher, 2011), pp. 167-194

[3] Rudge, A.W., Milne, K., Olver, A.D., et al: 'The handbook of antenna design' (Peter Peregrinus Ltd., 1983), vol. 2

[4] Jung, Y.B., Eom, S.Y., Jeon, S. I.: 'Novel antenna system design for satellite mobile multimedia service', IEEE Trans. Veh. Technol., 2010, 59, (9), pp. $4237-4247$

[5] Encinar, J.A., Zornoza, J.A.: 'Three-layer printed reflectarrays for contoured beam space applications', IEEE Trans. Antennas Propag., 2004, 52, (5), pp. $1138-1148$
[6] Padilla, P., Muñoz-Acevedo, A., Sierra-Castañer, M., et al.: 'Electronically reconfigurable transmit array at $\mathrm{Ku}$ band for microwave applications', IEEE Trans. Antennas Propag., 2010, 58, (8), pp. 2571-2579

[7] Ethier, J., Chaharmir, M.R., Shaker, J., et al: 'Development of novel low-cost reflectarrays [antenna applications corner]', IEEE Antennas Propag. Mag., 2012, 54, (3), pp. 277-287

8] Huang, J., Encinar, J.A.: 'Reflectarray antennas' (Ed. Wiley, 2007)

[9] Malfajani, S., Atlasbaf, Z.: 'Design and implementation of a broadband single layer circularly polarized reflectarray antenna', IEEE Antennas Wirel. Propag. Lett., 2012, 11, pp. 973-976

[10] James, I.R., Hall, P.S.: 'Handbook of microstrip antennas' (IEE Peregrinus, London, England, 1989), vol. 1, Chap. 10

[11] Tahseen, M.M., Kishk, A.A.: 'Ka-band circularly polarized high efficiency wide band reflectarray using cross bow-tie elements', Prog. Electromagn. Res., 2015, 153, pp. 1-10

[12] Masa-Campos, J.L., Casla-Sanz, E., Sierra-Pérez, M., et al.: 'Stacked circular patch antenna with dual right/left hand circular polarization for wideband applications in X band', Microw. Opt. Technol. Lett., 2009, 51, (6), pp. 1419 1424

[13] Chaharmir, M.R., Shaker, J., Cuhaci, M. et al.: "Circularly polarised reflectarray with cross-slot of varying arms on ground plane', Electron. Lett. $2002,38,(24)$, pp. $1492-1493$

[14] Ren, L.S., Jiao, Y.C., Li, F, et al.: 'A dual-layer T-shaped element for broadband circularly polarized reflectarray with linearly polarized feed', IEEE Antennas Wirel. Propag. Lett., 2011, 10, pp. 407-410

[15] Malfajani, R, Atlasbaf, $Z$ : 'Design and implementation of a broadband single layer circularly polarized reflectarray antenna', IEEE Antennas Wirel. Propag. Lett., 2012, 11, pp. 973-976

[16] Padilla, J.L., Padilla, P., Valenzuela-Valdés, J.F., et al.: "High-frequency radiating element and modified $3 \mathrm{~dB} / 90^{\circ}$ electronic shifting circuit with circular polarization for broadband reflectarray device cells', Electron. Lett., $2014, \mathbf{5 0}$ (15) pp. 1042-1043

[17] Tienda, C., Encinar, J.A., Arrebola, M., et al: 'Design, manufacturing and test of a dual-reflectarray antenna with improved bandwidth and reduced crosspolarization', IEEE Trans. Antennas Propag., 2013, 61, (3), pp. 1180-1190 